\title{
Influencia de la Institucionalización del adulto mayor en el desempeño de la memoria declarativa Visuo-Verbal asociada a contenido emocional ${ }^{1}$
}

Influence of the elderly's institutionalization in the performance of the visual - verbal declarative memory associated with emotional content

Influência institucionalização de pessoas idosas no desempenho de memória declarativa Visuo -Verbal associadocomconteúdo emocional

\author{
Jorge Alexander Ríos-Flórez ${ }^{2}$ \\ Jhonatan Alejandro Grajales-Ciro ${ }^{3}$ \\ Lina Yurley Valle 4 \\ Recibido: 22.06.2016 • Arbitrado: 24.10.2016 • Aprobado:31.10.2016
}

\begin{abstract}
${ }^{1}$ Artículoproductodel macroproyecto "Evaluación dela memoria declarativa asociada a contenido emocional en el envejecimiento normal y patológico”, formulado en la línea de Investigación Neuroenvejecimiento y Demencias, vinculada al Grupo de Estudio e Investigación en Neurociencias Hippocampus. Fecha de inicio del subproyecto Mayo de 2015; Fecha de finalización Mayo de 2016.
\end{abstract}

${ }^{2}$ Psicólogo, Universidad Pontificia Bolivariana; Magíster en Neuropsicólogía Clínica y Especialista en Evaluación y Diagnóstico Neuropsicológico, Universidad de San Buenaventura, Bogotá, D.C.; Director del Grupo de estudio e Investigación en Neurociencias Hippocampus; Docente pregrado y posgrado Facultad de Psicología y Ciencias Sociales, Tecnológico de Antioquia Institución Universitaria; Institución Universitaria Politécnico Grancolombiano, Medellín, Colombia; Jriosflo@poligran.edu.co, alexanderriosflorez@gmail.com.

${ }_{3}^{3}$ Psicólogo, Investigador asociado Grupo Hippocampus, Medellín, Colombia; Profesional en Diócesis de Caldas, Antioquia; joalgrci@hotmail.com.

${ }^{4}$ Psicóloga, Investigadora asociada Grupo Hippocampus, Medellín, Colombia; Profesional en Institución de Educación Técnica Laboral de Antioquia; valle216@hotmail.com. 


\section{Resumen}

La investigación buscó caracterizar la influencia de la institucionalización del adulto mayor en el desempeño de la memoria declarativa asociada a contenidos emocionales, participaron 40 adultos mayores; 20 institucionalizados y 20 no institucionalizados con edades entre $55^{-}$ 95 años, con condición par entre ellos. Empleando diseño descriptivo-correlacional, los resultados de la investigación arrojaron que las personas no institucionalizadas identifican mayores contenidos emocionales asociados a información almacenada en la memoria declarativa; por su parte los institucionalizados atribuyen mayores contenidos emocionales relacionados con sorpresa a la información previamente guardada, contrario a los no institucionalizados, quienes asociaron al recuerdo emociones negativas, como el miedo. El grupo de adultos institucionalizados evidenció diferencias significativas en relación a la información extraída de la memoria declarativa cuando se asociaba a contenidos emocionales con recobros deficientes de la información.

Palabras clave: Cognición, Memoria declarativa, Envejecimiento, Emociones, Adulto mayor, Institucionalización.

\section{Abstract}

The research looked for characterize the influence of the elderly's institutionalization in the performance of declarative memory associated with emotional content, in which 40 elderly participated; 20 institutionalized and 20 non-institutionalized with age ranges between 55 and 95 years old, including torque condition. Using descriptive-correlational design, the research results showed that the non-institutionalized people identify higher emotional content associated with the information stored in the declarative memory, meanwhile institutionalized people, attribute greater emotional content related to surprise to the previously stored information, contrary to the non-institutionalized ones, who associated with the memory negative emotions such as fear. The group of institutionalized adults showed significant differences in relation to the information extracted from the declarative memory when emotional content was associated with poor recoveries of information. 
Key Words: Cognition, Declarative memory, Aging, Emotions, Elderly, Institutionalization.

\section{Resume}

O estudoprocurou caracterizar a influência da institucionalização de idosos no desempenho da memória declarativa associadocomconteúdo emocional, participaram 40 idosos; 20 institucionalizados e 20 não institucionalizados comidade 55-95 anos, incluindocondiçãosemelhante. Usando um método descritivo-correlacional, os resultados da pesquisa mostraram que as pessoasnão institucionalizados identificar informaçõesconteúdo emocional maisassociadosarmazenados na memória declarativa, enquantoisso institucionalizado, atribuir maiorconteúdo emocional relacionado a surpreender, as informações salvas anteriormente, aocontrário da não institucionalizados, que associou as emoções negativas de memória, tais como o medo. O grupo de idosos institucionalizados apresentoudiferenças significativas emrelação à informação extraída a memória declarativa, quandoassociadocomconteúdo emocional comrecuperações pobres de informação.

Palavras chave:Cognição, Memóriadeclarative, Envelhecimento, Emoções, Terceiraidade, Institucionalização

\section{Introducción}

La vejez hace parte ineludible del proceso evolutivo en todos los seres humanos e inevitablemente en esta etapa, como en todas las del desarrollo, el cuerpo, la mente y sus funciones sufren cambios importantes, inherentes al estado de envejecimiento normal que desde la perspectiva de Losada (2004), constituye la vejez saludable, carente de enfermedad o de alteraciones biopsicosociales.

En la adultez se hace evidente el declive de algunas funciones físicas, psicológicas y sociales (Ardila, 2012). En las funciones físicas se incluyen también las cerebrales; a partir de los 60 años de edad el cerebro pierde volumen y peso, afectando en mayor medida a los lóbu- 
los frontales, temporales, parietales y la región parasagital, los menos afectados son la base del cerebro y los lóbulos occipitales, estos cambios morfológicos cerebrales provocan un enlentecimiento a nivel motor, sensorial, cognitivo (Dámaso \& Fernández, 2012). Lo que significa que este cambio cerebral afecta directamente las conductas físicas, que se evidencian en movimientos más lentos y menos finos, en cognición hay un evidente deterioro en la capacidad atencional, pero la queja más frecuente es la disminución en la retención de información, es decir lo concerniente a la memoria (Belsky, 2001; Hernández, Montañés, Gámez, Cano \& Núñez, 2007; Small, Gagnon \& Robinson, 2007; Binotti, Spina, De la Barrera \& Donolo, 2009).

El envejecimiento es en sí mismo un proceso evolutivo cuya calidad está directamente relacionada con la forma como el individuo satisface sus necesidades básicas a través de todo su ciclo vital (Sarabia, 2009). En esta etapa intervienen factores tanto fisiológicos como ambientales $\mathrm{y}$, por supuesto, componentes de tipo cultural que influyen en el ritmo del declive de las funciones físicas y cognitivas de cada sujeto, por ende, no se puede hablar de un deterioro igual en toda la población de adultos mayores, ya que se trata de un proceso dinámico con múltiples trayectorias (Losada, 2004).

Si bien a los adultos mayores en esta etapa de su vida se les dificulta su autonomía física, cabe resaltar que el déficit en la capacidad retentiva es lo que más afecta a esta población, déficit que se presenta aun sin que el adulto mayor padezca algún tipo de patología mental (Roman \& Sánchez, 1998; Demburg, Buchanan, Tranel \& Adolphs, 2003; Binotti et al., 2009).

\section{Referentes teóricos}

\section{Memorias y envejecimiento normal y patológico}

En relación a ello la memoria es precisamente aquel proceso mental producto de la actividad cerebral que nos permite registrar, almacenar y evocar toda la información que estuvo en la conciencia y que fue adquirida por medio de los sentidos (Baddeley, 1999; Bruning, Schraw \& 
Ronning, 2002; La Cruz, 2006), y puede medirse a través del reconocimiento de personas, palabras, situaciones y lugares (Rossatt, Chávez, Ribeiro \& Izquierdo, 1990). La memoria no es un sistema unitario, es un sistema variado que necesita la participación de distintas estructuras y circuitos cerebrales para su perfecto funcionamiento. Este planteamiento viene dado porque los sistemas varían de acuerdo a la duración de almacenamiento que van desde fracciones de segundo hasta un periodo de tiempo capaz de reseñar toda la vida de una persona (Blasco \& Meléndez, 2006), y es gracias a esta función de la memoria, que los individuos pueden desarrollar la capacidad de independencia, productividad y continuidad a lo largo de su existencia.

Particularmente, cuando se quiere avocar información de manera consciente es necesario la activación de los almacenes de memoria a largo plazo, específicamente la de tipo declarativa (Tómaz \& Costa, 2001). La memoria declarativa almacena información que se puede expresar de manera verbal, que puede ser evocada conscientemente; hechos, acontecimientos y estímulos determinados (Tómaz \& Costa, 2001; Mañeru, Junqué, Botet, Tallada, Segarra \& Narberhaus, 2002; Morgado, 2005; La Cruz, 2006).

Para que todo el procesamiento de la información se produzca de manera adecuada, desde el registro inicial hasta la reproducción de la misma, es necesario que otras funciones neuropsicológicas y numerosos circuitos del sistema nervioso central hagan su intervención y permanezcan sin ninguna alteración; es el caso de la atención, la percepción y el lenguaje mismo (Sánchez \& Pérez, 2008). La atención sirve como elemento activador de la percepción mediante operaciones de selección, distribución y mantenimiento de la actividad psicológica (Blasco \& Meléndez, 2006; Hernández et al., 2007).

En los adultos mayores la atención presenta un enlentecimiento significativo, su desempeño sufre algunas alteraciones con el paso de la edad que se evidencian en el pérdida de la exactitud en la detección de señales afectando el grado de vigilancia en las tareas que requieran especial atención (Hernández et al., 2007). 


\section{Influencia de las emociones sobre la cognición}

Otras funciones neuropsicológicas que tienen su papel en el procesamiento de la información y su adecuada evocación es la relación entre la cognición y la emoción, estos dos términos empezaron a aparecer juntos hace poco tiempo. A grandes rasgos, podríamos decir que surgen en los años 60 los primeros modelos cognitivos de la emoción en los que se empieza a suponer que hace falta cierta actividad cognitiva, para que se desarrolle una reacción emocional (Schachter\& Singer, 1962).

A las emociones se les conoce como estados perceptivos que generan sensaciones de placer o displacer dependiendo de la valoración cognitiva que se le dé a la información proveniente de una situación, cuya finalidad es la de movilizar la capacidad de reacción y adaptación del sujeto al medio (LeDoux, 1993; Lang, 1995; Aguado, 2002; Navarro, 2002; Randall, 2004; Chóliz, 2005).

La relación entre memoria y emoción radica precisamente en que la activación emocional o vivencias emocionales fuertes potencializan la elaboración de recuerdos que perduran en la memoria a largo plazo (Kazui, Mori, Hashimoto, Hiromi, Imamura, Tanimukai, Hanihara \& Cahill, 2000; Hamann, 2001; Aguado, 2002; Romeu, 2002; Kazui, Mori, Hashimoto \& Hirono, 2003; Botelho, Martinéz, Conde, Prada \& Becerra, 2004; Reisberg \& Heuer, 2004; Gordillo, Arana, Mestas, Salvador, Garcia, Carro \& Pérez, 2010).

De la relación mencionada anteriormente entre la memoria y la emoción, podría considerarse que finalmente la memoria emocional es el resultado de la adquisición, almacenamiento y reproducción de la activación emocional generada por medio de una experiencia de vida particular. La esfera emocional, al igual que las demás funciones neuropsicológicas, también muestra alteraciones con el paso de la edad, según lo referencian algunos estudios donde se pudo observar en los sujetos una disminución de las sensaciones de placer (Cahill \& McGaugh, 1995; Aguado, 2002). 


\section{Sustrato neuroanatómico y modificaciones funcionales tras el envejecimiento}

En todo el procesamiento de la información adquirida y almacenada en la memoria a largo plazo participan diferentes estructuras cerebrales como el lóbulo temporal (hipocampo, amígdala y corteza olfatoria), corteza prefrontal y núcleos del tálamo (Tirapú-Ustárroz \& Muñoz-Céspedes, 2005; Solis \& López, 2009). Algunos estudios realizados con animales y seres humanos han destacado precisamente la importancia del hipocampo en la memoria y el aprendizaje, se considera que de esta estructura depende incluso el registro inicial y consolidación de conocimientos explícitos y conscientes de experiencias vividas.

Las estructuras cerebrales implicadas directamente en la respuesta emocional son la corteza prefrontal y la amígdala, esta última es que la que más se relaciona con la emoción, pues cumple una función esencial en el procesamiento de las señales sensoriales, ya que recibe todas las proyecciones sensoriales, función que posiciona a esta estructura como la responsable de asociaciones entre los estímulos positivos o negativos. Esta red de influjos talámicos y corticales que llegan hasta la amígdala son los que permiten que se dote de un significado afectivo a las características estimulares (Sánchez \& Roman, 2004).

\section{Institucionalización como factor activador del deterioro neuropsicológico}

En consideración a lo anterior, uno de los factores que interfiere en el declive cognitivo y la evolución del mismo, es la condición de institucionalización; pese a que poco se ha abordado el tipo de influencia que representa y las consecuencias de la misma. En este sentido, Cerquera (2008) refiere que la condición de institucionalización del adulto mayor indica la incorporación del adulto a una institución encargada de sus cuidados y el aislamiento voluntario o no de su familia. Este abandono de su entorno familiar y social propio supone, sin duda alguna, una renuncia a su zona de confort, al contexto donde el adulto sabe moverse con facilidad y, muy seguramente, ha construido toda su his- 
toria personal; este cambio tiene un costo no solo físico sino también psicológico para el adulto mayor que, durante la adaptación al nuevo escenario, puede experimentar momentos de estrés y de depresión a raíz de las múltiples pérdidas: pérdida de la independencia, del contacto con sus familiares, desarraigo de los bienes, del rol social y de alguna actividad que lo hiciera sentir útil. Estas situaciones pueden acelerar, aún más, el declive de sus funciones físicas y cognitivas (Cardona, Estrada, Chavarriaga, Segura, Ordoñez \& Osorio, 2010).

Algunos estudios, realizados en esta misma población, han demostrado cómo las estructuras sociales, es decir, las redes de apoyo, son un potencial recurso para que los adultos mejoren su estado de salud y bienestar. Estas redes sociales como la familia, grupos de la tercera edad, grupos de deporte, de cultura, cumplen una función restauradora cuando la salud del adulto mayor está en riesgo, precisamente porque es en esta época de la vida cuando el individuo más necesita de su reconocimiento como ser humano, útil y productivo (Roca\& Pérez, 1999).

Teniendo en cuenta el interés de la relación entre la memoria y la emoción, algunos autores han realizado diferentes estudios acerca de este tema como Kensinger, Anderson, Growdon y Corkin (2004), quienes mostraron que tanto los sujetos jóvenes como los ancianos sanos, presentan mejor rendimiento para evocar recuerdos con contenido emocional que con contenido neutro. Sin embargo, los ancianos no muestran la misma facilitación del recuerdo en presencia de un contexto emocional, comparado con uno neutro.

Botelho de Oliveiraet al. (2004), en su estudio de la evaluación de la memoria declarativa asociada al contenido emocional (en una muestra colombiana con pacientes sanos) encontró que la memoria declarativa que hace parte de memoria a largo plazo puede ser potencializada tras una activación emocional causada por la vivencia de una situación con contenido emocional.

Bentosela y Mustaca (2005) mencionan que la reproducción de la información adquirida se hace fácil cuando esta está atravesada por estímulos o contextos emocionales. 
Para el año 2008 Botelho de Oliveira y sus colaboradores publicaron otro estudio sobre la evaluacion de la memoria declarativa asociada al contenido emocional, pero en pacientes lobectomizados, en donde se obtuvo como resultado que la memoria declarativa se puede potencializar por medio de situaciones que contengan emociones.

En el mismo año 2008, Conde, Prada, Martinez, Botelho y Becerra aseguran en su investigacion sobre la evaluacion de las manifestaciones autonomicas asociadas a la aplicación de una prueba auditivo visual de memoria emocional en humanos, que el grupo de participantes que habían presenciado la version emocional de la prueba $\mathrm{A}-\mathrm{V}$, habían mostrado mayor grado de alerta y desempeño en la capacidad de recordar en comparacion al grupo de participantes que presenciaron la versión neutra de la prueba.

Brochey Herrera (2011) refieren en su investigación, dirigida a describir la memoria de rostros y el reconocimiento emocional en adultos mayores institucionalizados y no institucionalizados sanos, que existen diferencias significativas entre los grupos respecto al reconocimiento de emociones, viéndose más afectado los adultos mayores institucionalizados.

En el año 2012 Gamboa y Vaquero pusieron en evidencia a través de su ejercicio investigativo con participación de adultos mayores institucionalizados de la ciudad de Bucaramanga y el municipio de Floridablanca, que los adultos mayores sometidos a la prueba auditivo visual con contenido emocional tuvieron una mayor potencialización de los recuerdos tal como en los estudios anteriores donde se ha aplicado la misma prueba.

Desde los referentes de la literatura científica y descriptivas del funcionamiento cognitivo en el envejecimiento, son diversos los aportes que apuntan a la influencia de las emociones en el contenido de la información a almacenar y recordar; sin embargo, pese a no haber un consenso en ello son escasos, más aún, los datos de investigaciones que apunten a la influencia de contextos de institucionalización sobre el deterioro y funcionamiento de las funciones cognitivas y la actividad cerebral en general, lo que propicia y pone de manifiesto la necesidad de ahondar en estas interrelaciones. 


\section{Metodología}

Esta investigación es de enfoque cuantitativo, con diseño descriptivo - correlacional; la selección de la muestra se realizó por modelo no probabilístico y voluntario (siguiendo los lineamientos de Hernández, Fernández \& Baptista, 2014).

\section{Medidas estadísticas}

Los datos obtenidos en la recolección de información producto del protocolo aplicado, se analizaron mediante estadística descriptiva-correlacional (Hernández et al, 2014); empleando el software estadístico SPSS versión 22, se ejecutó prueba de identificación de normalidad de los datos, de Kolmogorov-Smirnov con corrección de significación de Lilliefors; pruebas paramétricas a T de Student, no paramétricas a U de Mann-Whitney; coeficientes de correlación de Pearson y Spearman; frecuencias y descriptivos generales. Los análisis realizados buscan aumentar la seguridad y confiabilidad de los datos presentados, a partir de las medidas estadísticas idóneas según las características de la información cuantitativa procesada; así, en consideración a los resultados de la prueba de normalidad se ejecutaron los análisis paramétricos o no paramétricos según corresponda con el resultado estadístico inicial; de igual forma, aun cuando se ejecutó amplia cantidad de análisis estadísticos, se presentan aquí aquellos relevantes para los objetivos de la investigación, y las correlaciones pertinentes para las variables y criterios abordados.

\section{Sujetos}

La muestra total de 80 adultos mayores se divide en dos grupos: 40 adultos mayores institucionalizados y 40 adultos mayores no institucionalizados; sin alteraciones neuropsicológicas, mentales y/o físicas diferentes a las asociadas al envejecimiento normal; para la selección de la muestra no institucionalizada se tomó como criterio la condición para el adulto mayor institucionalizado, en relación a edad y género. Cada grupo de subdividió aleatoriamente y la mitad de cada muestra de adultos mayores presenciaron la prueba auditivo-visual de 
contenido neutro y la otra mitad presenciaron la prueba auditivo-visual de contenido emocional; a todos se les aplicó el protocolo restante de forma igual.

\section{Instrumentos}

El protocolo se elaboró y desarrolló teniendo en cuenta los test, sub test y pruebas seleccionadas; GDS: Escala de depresión geriátrica, por Yesavage y Sheikh en 1986, prueba de memoria declarativa Auditivo-Visual, versión colombiana, Botelho et al, (2004). Neuropsi: Evaluación neuropsicológica breve en español, por: Ostrosky, Ardila y Rosselli, (2000). The California verbal learning test: curva de memoria California, creada por Woods, Delis, Scott, Kramer, y Holdnack (2000). Test stroop: Test de colores y palabras Stroop elaborado por Charles Golden, en 2010. Trail Making Test: Test de trazos (TMT), creado por Reitan, en 1958 y el Test de reconocimiento de emociones en rostros (Baron-Cohen, Wheelwright \& Jolliffe, 1997).

\section{Procedimiento}

Para el desarrollo de las evaluaciones se realizaron tres sesiones con cada uno de los adultos mayores participantes, de una hora aproximadamente. Al inicio de cada una se buscó verificar el estado atencional y emocional del participante, y controlar las variables contextuales y ambientales que pudieran interferir en la aplicación de los test, seguido a ello se inició la aplicación de las pruebas que conformaron el protocolo distribuidas a lo largo de las 3 sesiones.

\section{Consideraciones éticas}

Los procedimientos realizados en la presente investigación se diseñaron de conformidad con las directrices estipuladas por el ministerio de salud nacional de Colombia (Resolución No 8430 de 1993), sobre la investigación con participantes humanos y el nivel de riesgo que implica. Por lo tanto, el estudio se cataloga como riesgo mínimo, debido a que no puso en riesgo la integridad física o psicológica de los parti- 
cipantes. Adicionalmente la investigación se ajustó a las disposiciones estipuladas por el código ético y deontológico del psicólogo (Ley 1090 de 2006), con relación a la protección de la identidad, buen nombre, participación voluntaria y fines estrictamente académicos- investigativos de los resultados de investigación. Así, la presente investigación se realizó bajo la aprobación de los participantes y su acudiente a través del consentimiento informado, previo a la realización del procedimiento de aplicación; se diligenció el consentimiento informado firmado por cada participante y representante legal. Para lo anterior, se les explicó en qué consistía el procedimiento y qué implicaciones tenía el hecho de aceptar participar en el mismo, el carácter voluntario de la participación y la posibilidad de abandono del estudio con previo aviso.

\section{Resultados}

Los análisis descriptivos de las muestras seleccionadas indican que la población se encuentra con una media de edad de 72.73 años (DE 10). Los test aplicados a valoración del estado cognitivo general (Neuropsi puntuación promedio 104, según intervalo de edad) y procesos atencionales (TMT A - B Y STROOP) refieren puntuaciones similares a nivel de la población general, indicando equivalencia entre grupos; la escala de depresión geriátrica (GDS) no reporta presencia de sintomatología depresiva al momento de la medición y aplicación de instrumentos. 
Influencia de la Institucionalización del adulto mayor en el desempeño de la memoria declarativa Visuo-Verbal asociada a contenido emocional

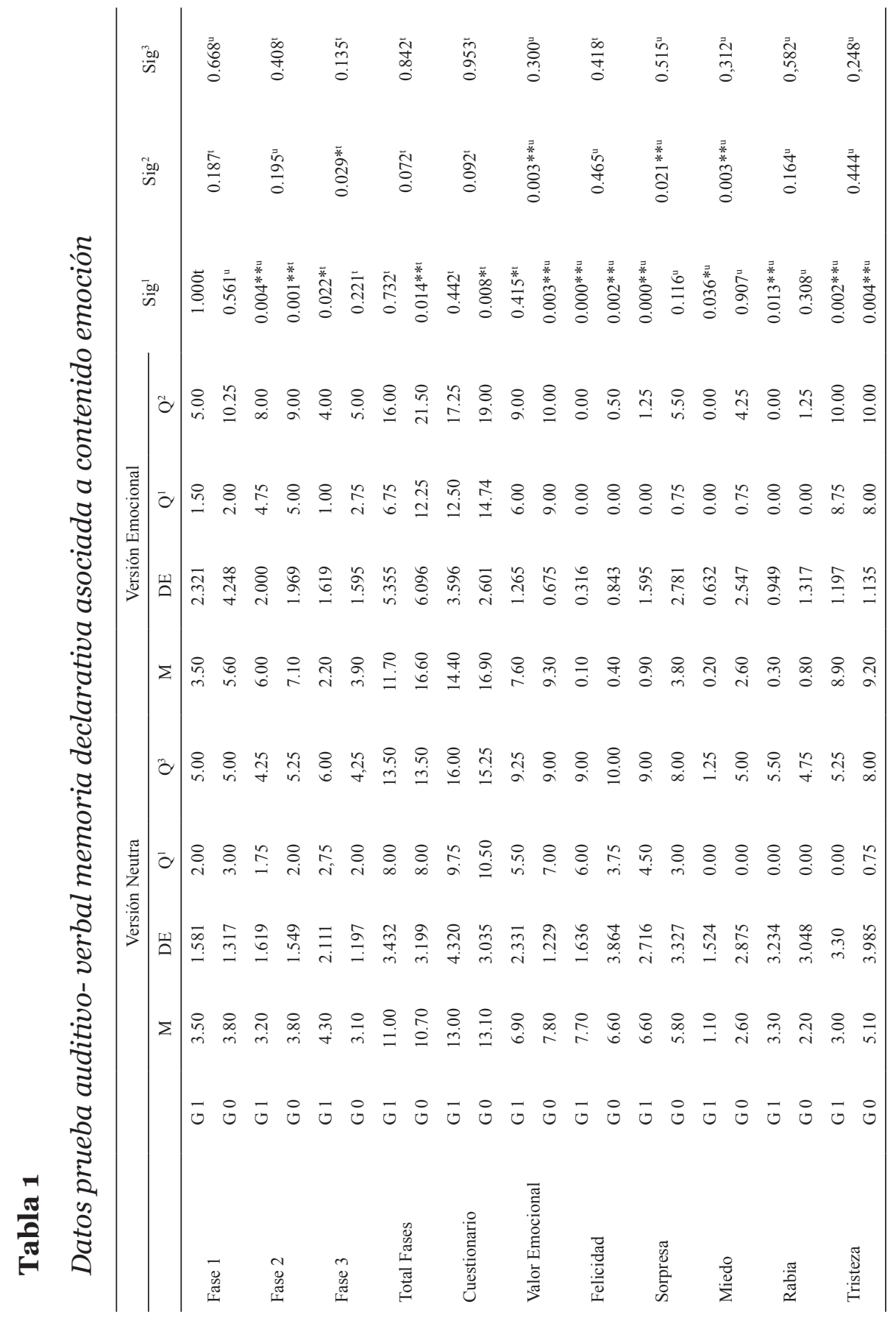


Nota: M: media; DE: desviación estándar; Q: cuartil; r: correlación; G o: grupo no institucionalizado; G 1: grupo institucionalizado; ${ }^{*} \mathrm{P} \leq 0.05 ;{ }^{*} \mathrm{P} \leq 0.01 ;$ t: T de Student; ${ }^{\text {: }}$ U de Mann-Whitney; Sig': análisis de significancia del grupo en relación a las versiones (Emocional-Neutra); $\mathrm{Sig}^{2}$ : análisis de significancia de la versión emocional en relación a los grupos; $\mathrm{Sig}^{3}$ : análisis de significancia de la versión neutra en relación a los grupos.

\section{Fuente: Autores}

Los análisis estadísticos presentes en la tabla 1 indican que para la versión de contenido neutro de la prueba de memoria declarativa no hay evidencias estadísticamente significativas entre los dos grupos, indicando esto que hay una cantidad de recuerdo similar y una valoración emocional similar entre ellos para esta variable, aun cuando la comparación de medias refiera puntuaciones superiores o inferiores a 2 o 3 puntos; sin embargo, se encuentra que para la versión emocional de la prueba, la fase 3 presenta datos significativos a nivel de $\mathrm{P} \leq \mathrm{0,05}$ sugiriendo una mayor taza de recuerdos asociada a la emoción en el grupo 1, es decir, el conformado por personas institucionalizadas. De igual forma, se encontraron diferencias significativas desde el punto de vista estadístico entre grupos para los criterios de sorpresa y miedo, así como para el valor emocional general asociado a la prueba. En relación a este último, el grupo de personas no institucionalizadas identifica mayor contenido emocional asociado a la información almacenada en la memoria declarativa, que el grupo de personas que sí se encuentran en una institución geriátrica. Por otra parte, los adultos mayores institucionalizados atribuyen mayores contenidos emocionales asociados con sorpresa a la información previamente guardada; contrario a ello es el grupo de no institucionalizados quienes en el contenido de la prueba asociaron al recuerdo mayores emociones relacionadas con el miedo.

De igual forma la tabla 1 permite identificar diferencias estadísticamente significativas a nivel de $\mathrm{p} \leq \mathrm{0,01}$ en relación a la versión presenciada intragrupo. En concordancia a esto se encuentra que para la fase 2, donde se encuentran las modificaciones emocionales de las dos 
versiones (emocional y neutra) se presentaron diferencias estadísticas entre quienes presenciaron una u otra versión a nivel de recuerdo de la información; así mismo, se encontró que para el total de fases el grupo no institucionalizado presenta diferencias significativas entre las versiones presenciadas: quienes vieron la versión emocional recuperaron mayor información en el análisis de comparación de medias intragrupo. De igual forma, se encontró que el recuerdo a largo plazo con el criterio cuestionario se ve favorecido en el grupo o en relación a la versión con contenido emocional en relación a la prueba para este grupo. Igualmente, se encuentran valoraciones emocionales asociadas al recuerdo con diferencias intragrupo a nivel de $\mathrm{p} \leq 0,05$ para el grupo 1 y de $\mathrm{p} \leq \mathrm{0,01}$ para el grupo 0 , siendo en ambos grupos una mayor valoración para quienes presenciaron la versión emocional; en relación a las emociones de sorpresa, miedo y rabia las diferencias intragrupo para la versión presenciada se encuentran significativamente en los adultos mayores institucionalizados asociando estas emociones a la información almacenada; en relación a la información asociada a contenidos de tristeza los análisis de ambos grupos refieren diferencias a nivel de $\mathrm{p} \leq \mathrm{O}$, 01 con mayor asociación de la información hacia la versión emocional de la prueba en un análisis intragrupo, tal como sucede con una valoración menor asociada a felicidad intragrupo según la versión presenciada y en los dos grupos con el mismo valor de significancia, lo anterior en relación al análisis de comparación de equivalencia de medias. 
Jorge Alexander Ríos-Flórez / Jhonatan Alejandro Grajales-Ciro / Lina Yurley Valle

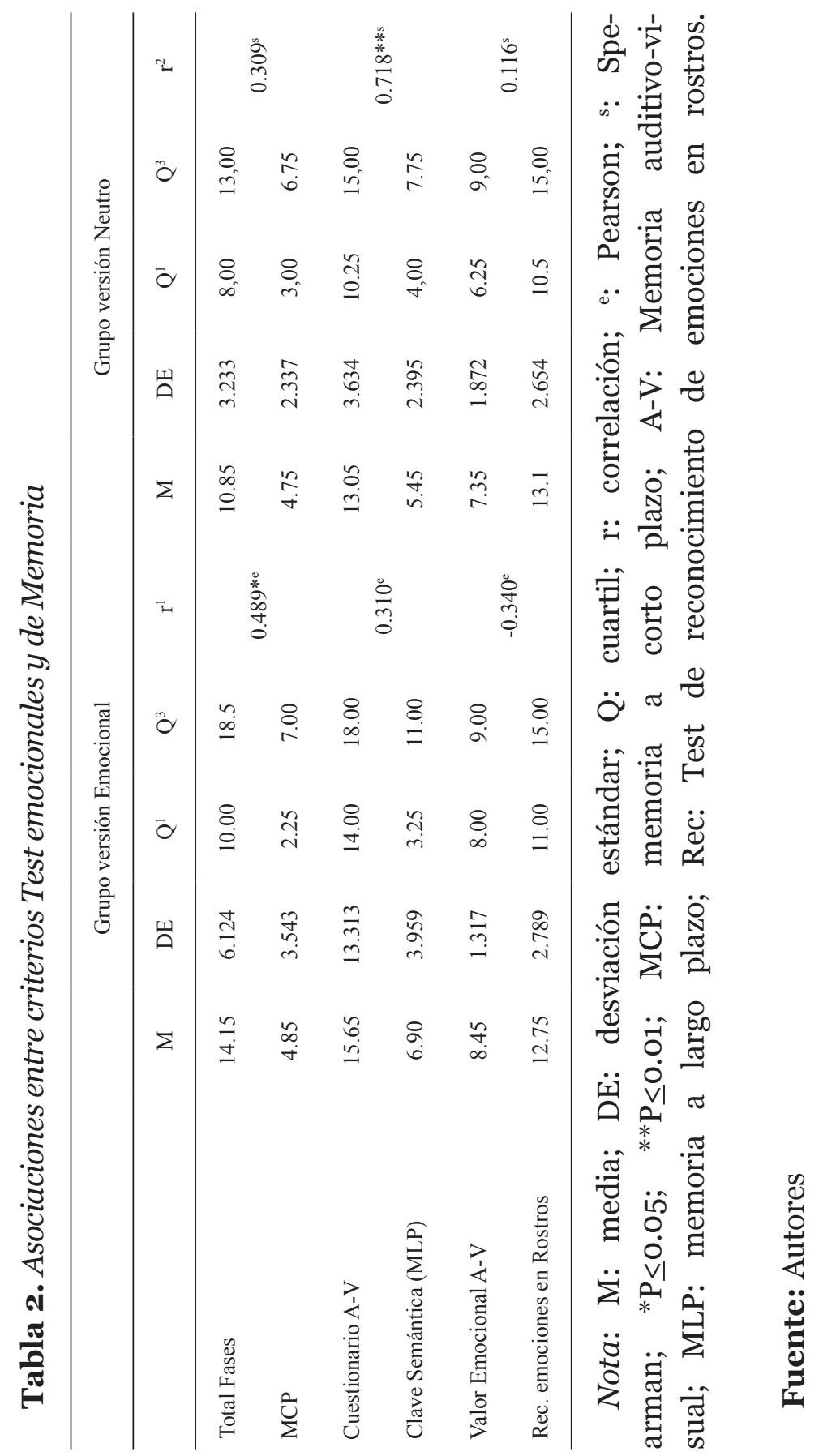


Por otra parte los análisis presentes en la tabla 2 refieren correlación positiva en los componentes de la memoria a corto plazo al asociar la puntuación total de las fases de la prueba auditivo visual de memoria declarativa con el apartado de memoria a corto plazo del test California para los adultos mayores que presenciaron la versión emocional en ambos grupos (institucionalizados y no institucionalizados). No así para quienes se les aplicó la versión neutra; contrario a ello, este último grupo a nivel de la memoria a largo plazo presenta correlaciones a nivel de $\mathrm{p} \leq 0.01$ entre el criterio de cuestionario de la prueba de memoria declarativa con contenido auditivo visual y el apartado de memoria a largo plazo con clave semántica del test California. De otro lado, no se encuentran correlaciones para ambos grupos y entre ambas versiones al asociar el valor emocional asignado a la información presentada en la prueba de memoria declarativa $\mathrm{A}-\mathrm{V}$ y el test de reconocimiento de emociones en rostros. Sin embargo, para los adultos mayores que presenciaron la versión emocional, aun cuando no significativa, la correlación entre estos criterios es inversamente proporcional.

\section{Discusión}

Los resultados producto de la investigación revelan datos variables que permiten coincidir por una parte y no concordar por otra con los planteamientos realizados por investigaciones previas, al analizar la influencia en la emoción sobre la memoria, y sobre estas la condición o no de institucionalización.

Por una parte, para el estudio aquí desarrollado, es posible coincidir con las afirmaciones producto de las investigaciones de Conde et al. (2008); Gamboa \& Vaquero (2012), cuando formulan que los participantes que recibieron información con carga emocional percibieron un mayor alertamiento y un mejor desempeño en la memoria para el recobro de información almacenada tanto para personas institucionalizadas como para personas no institucionalizadas. Lo anterior también se evidencia en las investigaciones desarrolladas por Botelho et al. (2008); Botelho et al. (2004); Satleret et al. (2007); Kazuiet al. (2000) y Kazuiet al. (2003). 
Desde aquí no es posible concordar con lo planteado por Kensingeret et al. (2004), al afirmar que los adultos mayores no muestran un mejor recobro de la información cuando se asocia a contenidos emocionales en comparación con contenidos neutros.

De otro lado, los resultados de la presente investigación permiten establecer que existen diferencias en la taza de evocación de la información a nivel de memoria declarativa cuando esta se asocia a contenidos emocionales y de relato espontáneo para los adultos mayores en condición de institucionalización; datos que coinciden con lo expuesto por Gamboa \& Vaquero (2012), quienes afirman mejor desempeño en los dispositivos de evocación libre de la información asociada a versiones emocionales en comparación con contenidos neutros.

En relación a la implementación de memoria declarativa asociada a contenido emocional Cahill \& McMaugh (1998) y Botelho et al. (2008), consideran que la fase 2 es la que presenta mayor contenido emocional de la historia, consideración que es homologable para los resultados de la investigación actual, tanto entre las dos versiones de la prueba para la fase 2 como de mayor sensibilidad bajo la condición o no de institucionalización. En este punto, Gamboa \& Vaquero (2012) refieren que el grupo emocional recordó más detalles de la historia que el grupo neutro en la fase 1 y que, por el contrario, no se encontraron diferencias significativas entre la fase 1 y 2 y en las fases 2 y 3 . Sin embargo, análisis desarrollados en la presente investigación permiten evidenciar que no existen diferencias en relación al recuerdo para la fase 1, ni entre versiones ni entre grupos; por otra parte, en relación a la fase 2, tal como se mencionó anteriormente, se haya sensibilidad para diferenciar la información recobrada y su asociación o no con contenidos emocionales; de otro lado, se encuentra que para la fase 3 hay diferencias significativas entre las dos versiones de la prueba al analizar el desempeño de los adultos mayores en condición de institucionalización, diferencias que no se presentan en los adultos no institucionalizados.

En la investigación desarrollada en 2008 por Botelho et al., se plantea que la tristeza es la emoción que más se asocia a la versión emocio- 
nal de la prueba; es preciso coincidir parcialmente con estos autores, considerando que la emoción de tristeza es asociada principalmente por los adultos mayores en condición de no institucionalización, seguido por la emoción de felicidad; sin embargo, bajo la condición de institucionalización la emoción que prima es la asociada con miedo y ausencia de asociaciones relacionadas con "rabia".

Autores como Broche \& Herrera (2011) refieren diferencias significativas entre los grupos respecto al reconocimiento de emociones, viéndose más afectados los adultos mayores institucionalizados; ante este planteamiento es posible coincidir encontrando que aun cuando la carga emocional favorece el recuerdo en ambos grupos, el grupo de institucionalizados presenta una menor cantidad de recuerdo de la información asociada a emociones primarias con características negativas, taza de recuerdo menor, en comparación con sus pares no institucionalizados.

En esta misma línea esta investigación encuentra una mayor asociación de emociones básicas como tristeza y miedo en información con carga emocional dentro de la memoria declarativa, y emociones como felicidad en información que carece de asociaciones emocionales, lo cual concuerda con lo expuesto por Gamboa \& Vaque (2012).

En este sentido, los resultados aquí analizados permiten establecer diversos planteamientos en relación a la influencia de la emoción y las características de dichas emociones al favorecer o no el recobro de la información contenida en los almacenes de largo plazo para memoria declarativa, desde lo cual se fomenta una discusión propositiva con los referentes de la literatura científica universal.

\section{Conclusiones}

Esta investigación revela aportes significativos al campo de la neuropsicología, la psicología en general y disciplinas de las neurociencias interesadas en conocimientos relacionados con el envejecimiento desde el punto de vista cognitivo conductual, emocional, y de funcionamiento cerebral, particularmente producto de este estudio en relación 
a las condiciones de institucionalización del adulto mayor y su influencia en procesos de memorias y emociones.

El contenido de la prueba auditivo -visual para evaluación de memoria declarativa asociada al contenido emocional es un instrumento eficaz en la evaluación de la relación entre estos dos componentes neurocognitivos; el grupo de pacientes institucionalizados evidenció diferencias significativas en relación a la información extraída de la memoria declarativa cuando se asociaba a contenidos emocionales.

La condición de institucionalización tiene influencia variable en relación a la forma en que emociones como miedo y sorpresa se asocian y favorecen el momento de la consolidación de la información en su paso a la memoria declarativa; los adultos mayores institucionalizados revelan menores asociaciones con sentimientos de tristeza y mayores en relación al miedo, lo que conlleva una mejor tasa de recuerdo; por el contrario los adultos mayores no institucionalizados asocian en su mayoría emociones de felicidad y/o tristeza al momento de formar sus memorias.

$\mathrm{Al}$ estar bajo condición de institucionalización los adultos mayores presentan una ausencia de asociar emociones relacionadas con sentimientos de "rabia" en sus experiencias biográficas y autobiográficas.

Los adultos mayores en condición de no institucionalización asocian a sus memorias de contenido declarativo una mayor gama de emociones entre las que destacan felicidad, sorpresa, miedo, rabia, y tristeza; por el contrario emociones como felicidad y tristeza son las que se encuentran relacionadas en los recuerdos declarativos de los adultos institucionalizados. Lo anterior, para ambos grupos, independientemente de los contenidos neutros o emotivos de la información.

En relación a los contenidos emocionales de la memoria declarativa, los adultos mayores institucionalizados presentan mayores asociaciones con contenidos emocionales en la información de su almacenamiento declarativo, principalmente emociones negativas.

La información dentro del almacenamiento de la memoria declarativa que carece de significancia emocional se discrimina y recuer- 
da de igual forma bajo la condición o no de institucionalización del adulto mayor.

Por otra parte, se encuentra de forma general asociación entre la memoria a corto plazo y la memoria a largo plazo en relación a los contenidos emocionales de cada almacén; de otro lado, la información de memoria a largo plazo, declarativa, que carece de asociaciones emocionales, requiere de claves semánticas para ser evocada por el adulto mayor, sin ser relevante la condición de institucionalización.

De forma indiferenciada las emociones influyen en el recuerdo de la información en los almacenes de memoria y su posterior evocación, ya sean asociadas a emociones básicas, complejas, positivas o negativas, que se pueden ver interferidas en su calidad y tasa de recuerdo de información bajo condiciones ambientales y/o personales.

En este punto, y partiendo de los referentes teóricos y de funcionamiento cognitivo obtenidos en la investigación, aun cuando los registros por neuroimágen no evidencien alteraciones estructurales en el cerebro del adulto mayor, áreas involucradas en el desarrollo de los procesos neuropsicológicos pueden verse comprometidas en funcionalidad.

En este sentido, el estudio y abordaje de patologías y temáticas como la expuesta aquí debe partir de la comprensión de la Neuropsicología y su interdisciplinariedad, es así que ésta debe considerarse como el estudio científico del pensamiento, la cognición y la conducta humana como producto de la actividad cerebral, que toma como base la funcionalidad normal y/o alteración del sistema nervioso y su organización anatómica e interacción fisiológica.

\section{Agradecimientos}

A los adultos mayores participantes de la investigación y a sus familias, así mismo, a las instituciones que aceptaron participar en la investigación y brindaron su tiempo para el desarrollo de la misma; de otro lado, a todos aquellos amigos y colaboradores que entienden la importancia de compartir el conocimiento e hicieron posible y accesible la búsqueda de literatura universal. 


\section{Referencias}

Aguado, L. (2002). Procesos cognitivos y sistemas cerebrales de la emoción.Revista Neurológica, 34 (12): 1161-1170. Recuperado de http://www.revneurol.com/

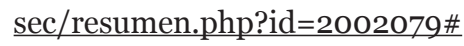

Ardila, A. (2012). Neuropsicología del envejecimiento normal. Revista Neuropsicología, Neuropsiquiatría y Neurociencias, 12 (1), 1-20. Recuperado de http:// neurociencias.udea.edu.co/revista/PDF/REVNEURO vol12 num1 5.pdf

Baron-Cohen, S., Wheelwright, S., Jollife, T. (1997). Is there a "lenguaje of the eyes"? Evidence from normal adults, and adults with autismo or Asperger Syndrome. Visual Cognition, 4: 311- 331. Recuperado de http://www.ncbi.nlm.nih.gov/ pubmed/9363580

Baddeley, A. (1999). Memoria humana. Teoría y Práctica.Madrid: Mc Graw Hill.

Belsky J. (2001). Psicología del envejecimiento. Madrid: Paraninfo.

Bentosela, M., Mustaca, A. (2005). Efectos cognitivos y emocionales del envejecimiento: aportes de investigaciones básicas para las estrategias de rehabilitación. Revista Interdisciplinaria. 22(2), 211-235. Recuperado de http:// www.redalyc.org/pdf/180/18022205.pd

Binotti, P., Spina, D., De la Barrera, M., Donolo, D. (2009). Funciones ejecutivas y aprendizaje en el envejecimiento normal. Estimulación cognitiva desde una mirada psicopedagógica. Revista Chilena de neuropsicología. 4 (2), 119-126. Recuperado de http://www.redalyc.org/pdf/1793/179314913005.pdf

Botelho, S., Albarracín, A., Chona, B., Conde, C.,Tomaz, C. (2008). Estudio correlacional entre memoria declarativa y una prueba psicométrica en una muestra de adolescentes escolares de la ciudad de Bucaramanga. Revista Colombia Médica. 39 (3), 60-70. Recuperado de http://www.bioline.org.br/ pdf?rco8069

Botelho, S., Martínez, L., Conde, C., Prada, E., Becerra, C. (2004). Evaluación de la memoria declarativa asociada al contenido emocional en una muestra colom- 
biana. Revista latinoamericana de psicología. 36 (2), 229-242. Recuperado de http://www.redalyc.org/pdf/805/80536204.pdf

Blasco, S., Meléndez, J. (2006). Cambios en la memoria asociados al envejecimiento. Rev. Geriatrika. 22 (5), 179-185. Recuperado de http://www.uv.es/melendez/ envejecimiento/memoriayvejez.pdf

Broche, P., Herrera, L. (2011). Memoria de rostros y reconocimiento emocional, particularidades en adultos mayores institucionalizados y que conviven con sus familiares. Revista Neuropsicologia. 3(3), 12-19. Recuperado de http://www. redalyc.org/pdf/4395/439542496002.pdf

Bruning, R., Schraw, G., Ronning, R. (2002). Psicología cognitiva e instrucción. España: Editorial Alianza editorial.

Cahill, L.,McGaugh, J. (1995). A novel demonstration of enhanced memory associated with emotional arousal. Conscious Cogn.,4:410-421. Doi: 10.1006/ ccog.1995.1048

Cardona, A., Estrada, R., Chavarriaga, L., Segura, A., Ordóñez, J., Osorio, J. (2010). Apoyo social dignificante del adulto mayor institucionalizado. Medellín, 2008. Revista Salud pública.12 (3), 414-424. Doi:10.159o/So12400642010000300007

Cerquera, A. (2008). Relación entre los procesos cognitivos y el nivel de depresión en los adultas mayores institucionalizadas, en el asilo San Antonio de Bucaramanga. Revista Universidad. Psychol, 7(1). 271-281. Recuperado de http:// www.scielo.org.co/pdf/rups/v7n1/v7n1a20.pdf

Conde, C., Prada, E., Martinez, L., Botelho, S.,Becerra, C. (2008). Evaluación de las manifestaciones autonómicas asociadas a la aplicación de una prueba auditivo-visual de memoria emocional en humanos. Revista Universidad Psichol. 7(1), 109-124. Recuperado dehttp://sparta.javeriana.edu.co/psicologia/publicaciones/actualizarrevista/archivos/o8-v7n1_condecortes_varios.pdf

Choliz, M. (2005). Psicología de la emoción: el proceso emocional. Universidad de 
Valencia.pp. 1-34. Recuperado de http://www.uv.es/choliz/Proceso\%20emocional.pdf

Dámaso, S., Fernández, C. (2012). Cambios cerebrales en el envejecimiento normal y patológico. Revista Neurop. 12 (1). 21-36. Recuperado de http://neurociencias.udea.edu.co/revista/?action $=$ resumen\&id $=180$

Demburg, N., Buchanan, T., Tranel, D. yAdolphs, R. (2003). Evidence for preserved emotional memory in normal older. The American Psychological Association. 3 (3), 239-253. Recuperado de http://www.ncbi.nlm.nih.gov/ pubmed/14498794

Gamboa, M., Vaquero, D. (2012). Evaluación de los efectos del contenido emocional en la memoria declarativa de tipo episódico en una muestra de adultos mayores institucionalizados.Medellín: Repositorio Universidad Pontificia Bolivariana.

Gordillo, F., Arana, J., Mestas, H., Salvador, J., Garcia, J., Carro, J., Pérez, H. (2010). Emoción y memoria de reconocimiento: la discriminación de la información negativa como un proceso adaptativo. Revista Psicothema. 22(4), 765-771. Recuperado de http://www.psicothema.com/pdf/3799.pdf

Golden, C. (2010). Stroop, Test de colores y palabras. Madrid: TEA Ediciones S.A. Hamann, S. (2001). Cognitive and neural mechanisms of emotional memory. Trends in Cognitive Sciences, 5, 394-400. Doi: 10.1016/S1364-6613(oo)01707-1

Hernández, R., Fernández, C., Baptista, P. (2014). Metodología de la investigación. 6ta edición. México: Ed. McGraw-hill.

Hernández, L., Montañez, P., Gámez, A., Cano, C., Núñez, E. (2007). Neuropsicología del envejecimiento normal. Revista De la asociación Colombiana de gerontología y geriatría .21(1) 992- 996. Recuperado de http://www.acgg.org.co/ descargas/revista-21-1

Kazui, H., Mori, E., Hashimoto, M., Hiromi, N., Imamura, T., Tanimukai, S., Hanihara, T., Cahill, L. (2000). Impact of emotional on memory. British Journal of 
Psychiatry. 177, 343-347. Doi: 10.1192/bjp.177.4.343

Kazui, H., Mori, E., Hashimoto, M., Hirono, N. (2003). Enhancement of declarative memory by emotional arousal and visual memory function in Alzheimer's disease. Rev. The Journal of Neuropsychiatry and Clinical Neurosciences. 15, 221-226. Doi: 10.1176/appi.neuropsych.15.2.221

Kensinger, E., Anderson, A., Growdon, J.,Corkin, S. (2004). Effects of Alzheimer disease on memory for verbal emotional informatión. Revista NeuroPsychologia. 42. 791-80o. Recuperado de http://web.mit.edu/bnl/pdf/Kensinger Neurop04.pdf

La Cruz, M. (2006). Estudio neurofisiológico de la memoria declarativa en el hombre. Tesis doctoral.pp. 1-185. Madrid, España: Universidad complutense de Madrid. Recuperado de http://biblioteca.ucm.es/tesis/med/ucm-t29247.pdf Losada, A. (2004). Las personas mayores. Observatorio de Personas Mayores, 1 , 557-577. Recuperado de http://envejecimiento.csic.es/documentos/documentos/losada-edadismo-01.pdf

Lang, P. (1995). The emotion probe. Studies of motivation and attention. Revista American Psychologist. 50, 372-385. Doi:10.1037/0003-066X.50.5.372

Le Doux, J. (1993). Emotional memory systems in the Brain. Behavior Brain Research, 58 (1-2), 69-79. Doi: 10.1016/0166-4328(93)90091-4

Ley 1090 de 2006. (2006).Código deontológico y bioético del psicólogo. Congreso de la república. Colombia.

Mañeru, C., Junqué, C., Botet, F., Tallada, M., Segarra, D., Narberhaus, A. (2002). Memoria declarativa y procedimental en adolescentes con antecedentes de asfixia perinatal. Revista Psicothema, 14, 463-468. Recuperado de http://www. redalyc.org/pdf/727/72714243.pdf

Morgado, I. (2005). Psicobiología del aprendizaje y la memoria: fundamentos y avances recientes. Revista Neurológica. 40 (5) 289-297. Recuperado de http:// www.revneurol.com/sec/resumen.php?id=2005004 
Navarro, X. (2002). Fisiología del sistema nervioso autónomo. Revista Neurológica, 35, 553-562. Doi: 10.5867/medwave.2001.04.3347

Ostrosky, F., Ardila, A., Rosselli, M. (2000). Evaluación neuropsicológica breve en español. México: Publingenio. S.A. de c.v.

Randall, D. (2004). Discovering the Role of the Adrenal Gland in the Control of Body Function. American Journal of Physiology - Regulatory, Integrative and Comparative Physiology, 287, 1007-1008. Doi: 10.1152/classicessays.00017.2004

Reitan, R. (1958) Validityn of the trail making test as an indicator of organic brain damage. Perceptual and motor Skills. 8, 271-276. Doi: 10.2466/PMS.8.7.271276

Reisberg, D. y Heuer, F. (2004). Remembering emotional events. En D. Reisberg y P. Hertel (Eds.): Memory and emotion. 3-41. New York: Oxford University Press.

Resolución No. 8430 de 1993.Normas científicas, técnicas y administrativas para la investigación en salud. Ministerio de salud (2003). Colombia

Roca, M. y Pérez, M. (1999). Apoyo social: su significado para la salud humana. Ciudad Habana. Editorial Félix Varela.

Rosatt, R., Chavés, M., Ribeiro, J., Izquierdo. I. (1990). The use of a new non-verbal test in the evaluation of recet memory. Brazilian Journal of medical and biological Research. 23(9), 805-809. Recuperado de http://europepmc.org/ abstract $/ \mathrm{med} / 2101320$

Roman, F., Sánchez, J. (1998). Cambios neuropsicológicos asociados al envejecimiento normal. Revista anuales de psicología. 14 (1). 27-43. Recuperado de http:// www.redalyc.org/pdf/167/16714104.pdf

Romeu, P. (2002). Recuerdo de imágenes emocionales y niveles de procesamiento. Revista Psicothema, 14(3), 591-596. Recuperado de nehttp://www.psicothema.com/psicothema.asp?id=770

Sánchez, G., Perez, M. (2008). Funcionamiento cognitivo en la vejez; atención y 
percepción en el adulto mayor. Cuba: Revista Cubana Médica, Gen integral. 24 (2); 1-7. Recuperado de://scielo.sld.cu/scielo.php?script=sci_arttext\&pid=So864-2125200800020001

Sánchez, J., Roman, F. (2004). Amígdala, corteza prefrontal y especialización hemisférica en la experiencia y expresión emocional. Revistas anales de psicología. 20 (2) 223-240. Recuperado de://www.um.es/analesps/v20/v20_2/o520_2.pdf

Sarabia, C. (2009). Envejecimiento exitoso y calidad de vida. Su papel en las teorías del envejecimiento. Revista Gerokomos. 20(4). 172-174. Recuperado de http://scielo.isciii.es/pdf/geroko/v2on4/breve1.pdf

Satler, C., Garrido, L., Sarmiento, E., Leme, S., Conde, C. y Tomaz, C. (2007). Emotionalarousalenhancesdeclarativememory in patientswithAlzheimer'sdisease. Acta Neurológica Scandinavica. 116 (6). 355-36o Doi: 10.1111/j.160o0404.2007.00897.x

Schachter, S., Singer, J. (1962). El enfoque cognitivo, la teoría. Revista española de neuropsicología. (6),53-73. Recuperado de https://dialnet.unirioja.es/descarga/articulo/2011686.pdf

Solis, H., López, E. (2009). Neuro- anatomía funcional de la memoria. Archivo Neurociencias. México. 14(3), 176-187. Recuperado de http://www.medigraphic. com/pdfs/arcneu/ane-2009/aneo93f.pdf

Small, B., Gagnon, E., Robinson, B. (2007). Early identification of cognitive deficits: Preclinical Alzheimer's disease and mild cognitive impairment. Revista. Geriatrics,62, 19-23. Recuperado de http://www.ncbi.nlm.nih.gov/ pubmed/17408315

Tirapu-Ustárroz, J. y Muñóz-Céspedes, J. (2005). Memoria y funciones ejecutivas. Revista neurológica. 41 (8) 475-484. Recuperado de http://www.neurologia.

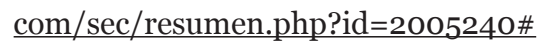

Tomaz, C. y Costa, J. (2001) Neurociência y memória. Revista Humanidades. Brasil- 
ia.48. 145-160. Recuperado de: repositorio.unb.br/.../1/2006_Paola\%2oRabello\%2oVieira.pdf

Yesavage, J., Sheikh, J. (1986). Geriatric Depression Scale, GDS. Clinical Gerontology: A guide to assessment and intervention. 165-173, NY: The Haworth press.

Woods, S., Delis, D., Scott, J., Kramer J. y Holdnack, J. (2006). The California verbal learning test- second edition: test-retest reliable change indices for the standard and alternate forms. Archives of Clinical Neuropsychology. V. 21, 413-420.Doi: 10.1016/j.acn.2006.06.002 